\title{
LABORATORY DETERMINATION OF LANDÉ FACTORS FOR THE MOLECULAR RADICAL FEH
}

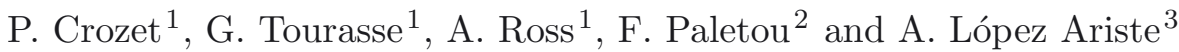

\begin{abstract}
We report laboratory measurements of the Zeeman response of lines in the $0-0$ Wing-Ford band of the F-X system $(\lambda \sim 1 \mu \mathrm{m})$ of $\mathrm{FeH}$, measured in magnetic fields $0.3-0.5$ Tesla. New Landé factors are used to deduce the magnetic field in sunspots from Stokes V profiles recorded at the solar telescope THEMIS. The magnetic field deduced from atomic lines ( $\mathrm{Ti}, \mathrm{Fe}$ ) is slightly higher than that found from $\mathrm{FeH}$.
\end{abstract}

\section{Introduction}

Zeeman-broadened lines in the near IR $F^{4} \Delta \leftarrow X^{4} \Delta$ (Wing-Ford) band of FeH have been proposed (Afram et al. 2008) as possible magnetic probes of cool stellar atmospheres. These $\mathrm{FeH}$ transitions have been observed and interpreted in sunspot spectra (Harrison \& Brown 2008), and in the spectra of type-L brown dwarfs (Shulyak et al. 2010). Reliable laboratory data are essential if such spectra to be interpreted correctly, because molecular Landé factors for transition-metal containing radicals are notoriously difficult to model and predict. The breakdown of the Born-Oppenheimer approximation, separating rotational, vibrational and electronic wavefunctions, results in apparent variations in the coupling schemes for molecular angular momenta. This situation was described (Harrison \& Brown 2008) in a paper listing empirical g-factors for selected lines of the Wing-Ford transition in FeH: the Landé factors were found to be intermediate between Hund's case (a) and case (b) values. Laboratory spectra of this system allow us to improve the effective Landé factors $g_{J}$ for the excited electronic state.

\footnotetext{
${ }^{1}$ LASIM Laboratoire de Spectrométrie Ionique et Moléculaire - UMR 5579 CNRS \& Université Lyon 1, 43 Bd. du 11 novembre 1918, 69622 Villeurbanne, France

2 IRAP Institut de Recherche en Astrophysique et Planétologie, UMR 5277 Université de Toulouse \& Observatoire Midi-Pyrenées (CNRS), 14 avenue E. Belin, 31400 Toulouse, France 3 THEMIS CNRS UPS 853 C/O IAC, via Lactea s/n, 38205 La Laguna, Tenerife, Spain
} 


\section{Laboratory experiment}

FeH is produced in a sputtering source (Vallon et al. 2009) using a DC discharge in a continuous flow of $10 \% \mathrm{H}_{2}$ in Ar, operating at pressures of the order of 1 Torr. A locally homogeneous field is created with a magnetic circuit based on a NdFeB permanent magnet. A tunable single-mode Ti:sapphire laser (Sirah Matisse TS) probes the molecular flux in near IR absorption bands $\left(9940-10070 \mathrm{~cm}^{-1}\right)$ in the $F^{4} \Delta_{\Omega} \leftarrow X^{4} \Delta_{\Omega}$ system at a chosen distance from the center of the magnetic circuit, allowing a range of magnetic fields to be investigated. Laser-induced fluorescence (a priori from FeH) is detected on a low-noise InGaAs detector, with simultaneous acquisition of Fabry-Pérot fringes and an $\mathrm{Rb}_{2}$ reference spectrum (Ross et al. 2010) for calibration purposes. Magnetic fields are readily calibrated to $0.5 \%$ accuracy by recording the response of Ar I lines at 10958.339 and $10351.501 \mathrm{~cm}^{-1}$ (Ralchenko et al. 2008), that are easily measured in the same discharge. We first recorded survey scans at zero field, to establish lambdadoubling patterns unresolved in earlier work (Dulick et al. 2003; Phillips et al. 1987), then in magnetic fields up to $0.5 \mathrm{~T}$, for a selection of lines with $J \leq 8.5$ accessible with our laser, working at a Doppler-limited resolution of $0.02 \mathrm{~cm}^{-1}$. Spectra are illustrated in Figure 1. Effective Landé factors $g_{J}$ were obtained by fitting measured wavenumbers to energy expressions for both electronic states

$$
E\left(J, \text { parity }, \nu, M_{J}\right)=E_{0}(J, \text { parity }, \nu)+\mu_{B} B g_{J} M_{J}
$$

where $\mu_{B}$ is the Bohr magneton $\left(0.4669 \mathrm{~cm}^{-1} / \mathrm{T}\right)$ and $B$ is the magnetic field (Tesla). The Landé factor $g_{J}$ is given in pure Hund's case (a) by

$$
g_{J}=\Omega \frac{g_{L} \Lambda+g_{S} \Sigma}{J(J+1)}
$$

We took literature values (Brown et al. 2006) for $g_{J}$ in the $X^{4} \Delta$ state, as the ground state parameters have been accurately established from laser magnetic resonance spectroscopy, and determined $g_{J}^{\prime}$ for the upper state. When rotational structures were insufficiently resolved to identify individual transitions, we obtained (less well-defined) $g_{J}^{\prime}$ by matching experimental and modelled profiles, assuming a Hönl-London $\mathcal{S}\left(M^{\prime}, M^{\prime \prime}\right) \times \mathcal{S}\left(J^{\prime}, J^{\prime \prime}\right)$ intensity distribution (following Berdyugina et al. 2002) amongst the $M_{J}$ components. Results are reported in Table 1, together with ground state $g_{J}$, and the values calculated for pure Hund's case coupling, for sake of comparison. At present, each $g_{J}^{\prime}$ value is determined from a single $\mathrm{Q}$ or $\mathrm{R}$ line in the spectrum. Work is in progress to enlarge the data set. The differences between the $g_{J}^{\prime}$ values we have obtained and those derived by Harrison \& Brown in 2008 (Harrison \& Brown 2008) using data taken from the (less well-resolved) sunspot Atlas published in 1998 (Wallace et al. 1998) are small but significant, as shown by the patterns illustrated in Figure 1b, where we present the two calculated profiles above one of the laboratory spectra. 
(b)
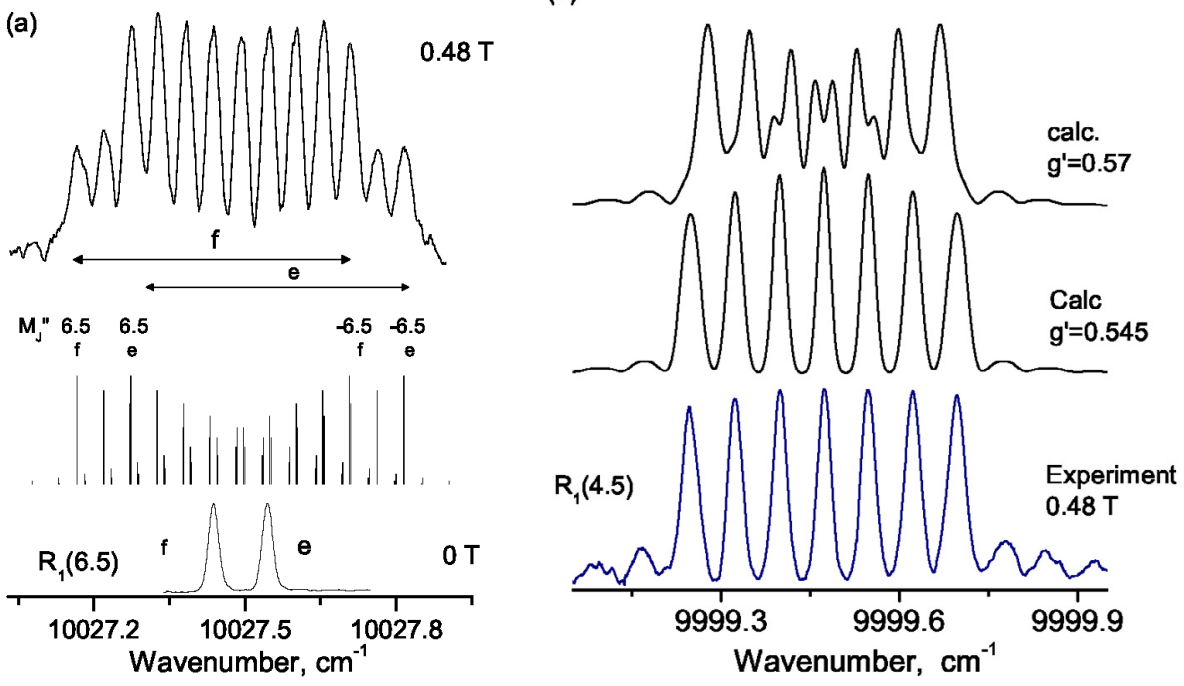

Fig. 1. Detail of two $R_{1}$ lines in the $0-0$ band. (a) $\mathrm{R}(6.5)$ line recorded at $B=0$ and $0.48 \mathrm{~T}$, showing the effect of $\Lambda$-doubling and Zeeman patterns selecting $\Delta M_{J}=$ +1 transitions. The stick spectrum shows calculated positions and intensities for individual $M_{J}$ components. (b) Observed and calculated profiles for $\mathrm{R}(4.5)$ at $0.48 \mathrm{~T}$, using $g_{J^{\prime}=5.5}^{\prime}$ values from Harrison \& Brown (upper trace) or from this work (middle trace).

Table 1. Some $g_{J}$ Landé factors for $\mathrm{v}=0$ in the $X^{4} \Delta$ and $F^{4} \Delta$ states of FeH. ${ }^{1}$ Taken from Brown et al. (2006); ${ }^{2}$ Taken from Harrison $\mathcal{E}$ Brown (2008).

\begin{tabular}{|c|c|c|c|c|c|c|}
\hline$J$ & parity & $\begin{array}{c}g_{J}\left(X^{4} \Delta^{7 / 2}\right) \\
\mathrm{LMR}^{1}\end{array}$ & $\begin{array}{r}g_{J}( \\
\text { this work }\end{array}$ & $\begin{array}{l}\left.\Delta_{7 / 2}^{4}\right) \\
\text { sunspot data }{ }^{2}\end{array}$ & $\begin{array}{l}\text { Hund's } \\
\text { case(a) }\end{array}$ & $\begin{array}{l}\text { Hund's } \\
\text { case(b) }\end{array}$ \\
\hline \multirow[t]{2}{*}{3.5} & $\mathrm{e}$ & $1.24802(4)$ & $1.130(6)$ & \multirow[t]{2}{*}{1.125} & \multirow[t]{2}{*}{1.111} & \multirow[t]{2}{*}{1.238} \\
\hline & $\mathrm{f}$ & $1.24795(3)$ & $1.128(6)$ & & & \\
\hline \multirow[t]{2}{*}{4.5} & e & $0.90284(3)$ & $0.749(2)$ & \multirow[t]{2}{*}{0.75} & \multirow[t]{2}{*}{0.707} & \multirow[t]{2}{*}{0.889} \\
\hline & $\mathrm{f}$ & $0.90263(2)$ & $0.749(2)$ & & & \\
\hline \multirow[t]{2}{*}{5.5} & $\mathrm{e}$ & $0.71110(3)$ & $0.545(3)$ & \multirow[t]{2}{*}{0.57} & \multirow[t]{2}{*}{0.489} & \multirow[t]{2}{*}{0.691} \\
\hline & $\mathrm{f}$ & $0.71053(2)$ & $0.545(3)$ & & & \\
\hline \multirow[t]{2}{*}{6.5} & $\mathrm{e}$ & $0.59109(8)$ & $0.423(5)$ & \multirow[t]{2}{*}{0.43} & \multirow[t]{2}{*}{0.359} & \multirow[t]{2}{*}{0.564} \\
\hline & $\mathrm{f}$ & $0.59015(8)$ & $0.421(5)$ & & & \\
\hline \multirow[t]{2}{*}{7.5} & $\mathrm{e}$ & $0.50954(9)$ & $0.340(2)$ & \multirow[t]{2}{*}{0.36} & \multirow[t]{2}{*}{0.274} & \multirow[t]{2}{*}{0.476} \\
\hline & f & $0.50791(7)$ & $0.340(2)$ & & & \\
\hline
\end{tabular}

\section{Sunspot spectra}

Some sunspot spectra were recorded at THEMIS in July 2011, using polarimetric techniques to record Stokes profiles in the 1 micron region, at an effective resolution 
estimated at $0.07 \mathrm{~cm}^{-1}(70 \mathrm{~m} \AA)$. The spectra show strong $\mathrm{Fe}$ and $\mathrm{Ti}$ atomic absorptions, and (much) weaker profiles from FeH lines, as illustrated in Figure 2. Using parameters from Table 1, we can deduce the magnetic field from these spectra. We find $\mathrm{B}=0.25(1) \mathrm{T}$ from the atomic lines, and $\mathrm{B}=0.22(1) \mathrm{T}$ from the $\mathrm{Q}_{1}(3.5)$ and $\mathrm{Q}_{1}(4.5) \mathrm{FeH}$ lines. We noticed distinctly different lineshapes and widths, with the atomic lines showing a significant Lorentzian component, while the molecular ones were apparently pure Gaussian.

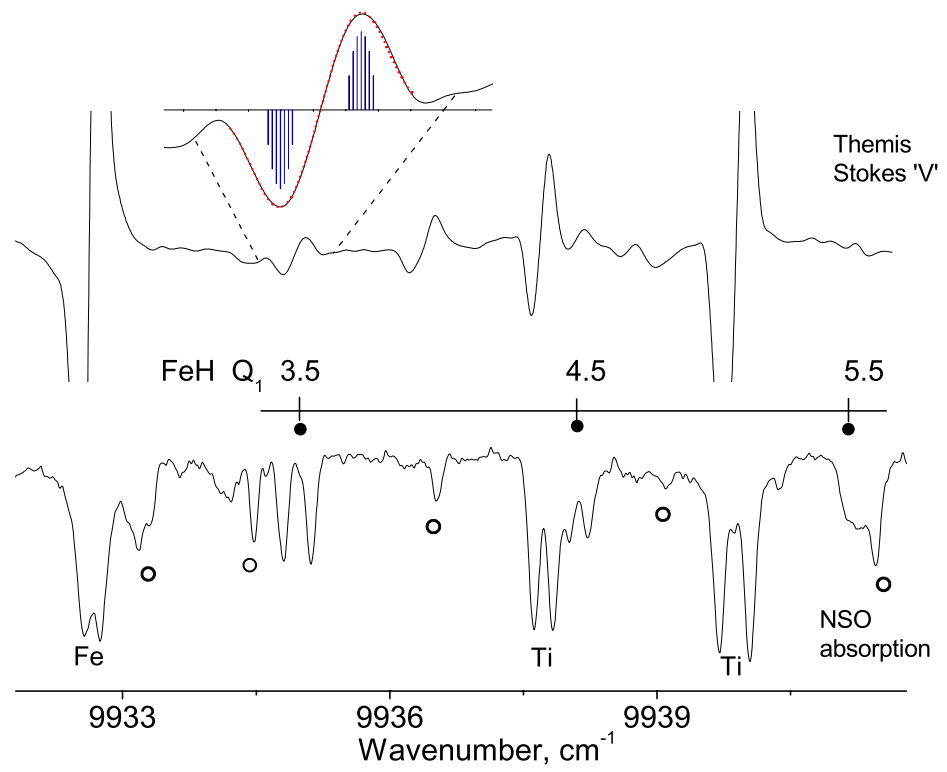

Fig. 2. Sunspot spectra, recorded at the solar telescope THEMIS (upper trace, showing Stokes V profiles) and at Kitt Peak (lower trace, absorption spectrum from Wallace et al. 1998). Filled circles indicate transitions from the lowest spin component, $\mathrm{F}_{1}\left(F^{4} \Delta_{7 / 2}-\right.$ $\left.X^{4} \Delta_{7 / 2}\right)$. Open circles indicate $\mathrm{FeH}$ transitions from the higher spin components. The inset shows a profile fit to $\mathrm{Q}_{1}(3.5)$, with a stick spectrum showing the underlying individual $M_{J}$ components.

\section{Conclusions}

Earlier work at Arizona State University (Harrison et al. 2008) used a cold molecular beam source to probe just two lines, $\mathrm{R}(3.5)$ and $\mathrm{Q}(3.5)$, in the (1-0) band of the $F^{4} \Delta_{7 / 2}-X^{4} \Delta_{7 / 2}$ system in $\mathrm{FeH}$. We have extended measurements to higher $J^{\prime \prime}$ and established effective Landé factors for several rotational levels in the $\mathrm{v}^{\prime}=0$ of the $F^{4} \Delta_{7 / 2}$ state. Most of our measurements still relate to the $\Omega=7 / 2$ spin-orbit component, as this has the highest population distribution with our source, which operates around $650 \mathrm{~K}$. We note that one of the conclusions in a recent paper (Shulyak et al. 2010) was that magnetic fields (3-4 kGauss) 
in selected M-dwarf spectra derived from FeH lines were 15-30\% lower than the fields deduced from atomic Fe I lines. Our analysis of recent sun-spot spectra tend to confirm a difference in magnetic field response determined from atomic and molecular signatures. Molecules are presumably formed at different altitudes, in relatively cooler environments. Inversion of the observational spectra requires further work to take into account the effects of turbulence on the lineshapes, and still more complications will arise with rotational effects in dwarf-star spectra, as discussed by (Wende et al. 2009).

We acknowledge financial support from the French National Research Agency (grant ANR 08-BLAN-0017), and from the Programme National de Physique Stellaire (2010) (INSU-CNRS).

\section{References}

Afram, N., Berdyugina, S.V., Fluri, D.M., Solanki, S.K., \& Lagg, A., 2008, A\&A, 482, 387

Berdyugina, S.V., \& Solanki, S.K., 2002, A\&A, 385, 701

Brown, J.M., Korsgen, H., Beaton, S.P., \& Evenson, K.M., 2006, J. Chem. Phys., 124, 234309

Dulick, M., Bauschlicher, C.W., Burrows, A., Sharp, C.M., Ram, R.S., \& Bernath, P., 2003, ApJ, 594, 651

Harrison, J.J., \& Brown, J.M., 2008, ApJ, 686, 1426

Harrison, J.J., Brown, J.M., Chen, J., Steimle, T.C., \& Sears, T.J., 2008, ApJ, 679, 854

Phillips, J.G., Davis, S.P., Lindgren, B., \& Balfour, W.J., 1987, ApJS, 65, 721

Ralchenko, Y., Kramida, A.E., Reader, J., \& NIST-ASD-Team, 2008, NIST Atomic Spectra Database, http://physics.nist.gov/asd3

Shulyak, D., Reiners, A., Wende, S., Kochukhov, O., Piskunov, N., \& Seifahrt, A., 2010, A\&A, 523, A37

Ross, A.J., Bertrand, V., Harker, H., \& Crozet, P., 2010, J. Mol. Spectrosc., 264, 78

Vallon, R., Ashworth, S.H., Crozet, P., et al., 2009, J. Phys. Chem. A, 113, 13159

Wallace, L., Livingstone, W. Bernath, P., \& Ram, R.S., 1998, N.S.O. Technical Report 1998-002, Available online ftp://nsokp.nso.edu/pub/atlas/spot3alt

Wende, S., Reiners, A., \& Ludwig, H.G., 2009, A\&A, 508, 1429 